\title{
Case Report/Series
}

\section{Multifocal recurrence of medulloblastoma: a long follow-up case study}

\author{
Benny Zulkarnaien, ${ }^{1}$ Edwin Suharlim, ${ }^{1}$ Eka Susanto, ${ }^{2}$ Soehartati Argadikoesoema Gondhowiardjo ${ }^{3}$
}

Check for updates

pISSN: 0853-1773 - elSSN: 2252-8083 https://doi.org/10.13181/mji.cr.193480 Med J Indones. 2020;29:93-9

Received: January 10, 2019 Accepted: August 24, 2019

\section{Authors' affiliations:}

${ }^{1}$ Department of Radiology, Faculty of Medicine, Universitas Indonesia, Cipto Mangunkusumo Hospital, Jakarta, Indonesia, ${ }^{2}$ Department of Anatomical Pathology, Faculty of Medicine, Universitas Indonesia, Cipto Mangunkusumo Hospital, Jakarta, Indonesia, ${ }^{3}$ Department of Radiation Oncology, Faculty of Medicine, Universitas Indonesia, Cipto Mangunkusumo Hospital, Jakarta, Indonesia

\section{Corresponding author:}

Edwin Suharlim

Department of Radiology, Faculty of Medicine, Universitas Indonesia, Cipto Mangunkusumo Hospital, Jalan Diponogoro No. 71, Kenari, Senen, Central Jakarta 10310, DKI Jakarta, Indonesia

Tel/Fax: +62-21-3910462

E-mail: edwin_suharlim@yahoo.co.id

\begin{abstract}
Medulloblastoma is the most common malignant brain tumor in pediatric patients. Leptomeningeal dissemination often occurs in the spinal canal and rarely into the supratentorial as an extra-axial lesion. This study followed an 8-year progression of multifocal medulloblastoma recurrence as supratentorial metastasis that mimics an intra-axial mass of the temporal lobe. Pathologic examination of the temporal mass showed densely packed small round blue cells that are distributed in a ribboning pattern with areas of necrosis, rosette formation, and mitosis. Ki-67 immunohistochemical staining of the tumor showed a high proliferation index of $40 \%$ and revealed positive results for synaptophysin and negative results for glial fibrillary acidic protein and $\beta$-catenin. A long follow-up is essential to diagnose the recurrence and elucidate the progression of medulloblastoma.
\end{abstract}

KEYWORDS intra-axial mass, long follow-up, medulloblastoma, recurrence
Medulloblastoma, an aggressive tumor occurring in the cerebellum, is the most common neurological malignant neoplasm in children. ${ }^{1}$ Medulloblastoma recurrences are common within 2 years despite adequate multi-modality therapy of surgical resection, radiation, and chemotherapy. ${ }^{2}$ Leptomeningeal dissemination into the spinal canal commonly occurs because of the proximity between medulloblastoma and the fourth ventricle. However, supratentorial seeding rarely develop in areas with close contact to the cerebrospinal fluid. ${ }^{3}$

This report presents an 8-year follow-up of the medulloblastoma case of an 18-year-old male with multifocal recurrences. To the best of our knowledge, this study was one of the few to conduct a long follow-up imaging report on medulloblastoma. It describes the comprehensive imaging of primary cerebellar mass, spinal and supratentorial recurrences, 
disease progression that mimics intra-axial mass, and therapeutic response to chemotherapy and radiation.

\section{CASE REPORT}

An 18-year-old male initially presented with a chief complaint of prolonged headache. His headaches worsened progressively over the last 2 months, especially at the right side, accompanied by nausea and vomiting. Weakness, instability, fever, or previous symptoms of prolonged headache were not recorded before admission. Upon physical examination, neither neurological deficit nor vital sign abnormality was found.

Contrast magnetic resonance (MR) of the brain in the first meeting (Figure 1) revealed posterior fossa mass of the cerebellar vermis with heterogeneous enhancement. Single-voxel MR spectroscopy (MRS) of the mass indicated elevated choline (Cho) peak of 158,158 units and a low N-acetyl-aspartate (NAA) peak of 83,460 . MRS of the normal cerebellum indicated a Cho peak of 40,469 and an NAA peak of 122,304. The Cho/NAA ratio of the mass was 1.89, whereas that of the normal cerebellum parenchyma as seen in Figure 1 was 0.33 .

The patient declined surgical resection of the vermis and chose non-invasive treatment because of financial reasons. At the third month, the patient underwent stereotactic radiotherapy (SRT) of 63.6 Gy directed to the cerebellar vermis for 7 weeks. Mass volume reduced considerably after 1 month of radiotherapy and disappeared 5 months after completing SRT (Figure 2). Diffusion-weighted imaging (DWI) and apparent diffusion coefficient (ADC) map confirmed no pathological diffusion restriction of the cerebellar vermis.

Subsequent follow-up of brain and spine MR was performed at the $12^{\text {th }}$ month after disappearance of the cerebellar vermis tumor. Brain and spinal MR (Figure 3 a-d) showed vividly enhancing suprasellar mass, accompanied by smaller lesion frontal interhemispheric sulci. No lesion was found in the cerebellar vermis tumor bed. Spinal MR showed enhanced intradural extramedullary lesion in $\mathrm{C}_{4}-\mathrm{C}_{5}, \mathrm{C}_{2}-\mathrm{C}_{3}, \mathrm{~L} 4$, and $\mathrm{S}_{1}-\mathrm{S}_{2}$ (not shown). Lumbar puncture was then performed for cerebrospinal fluid (CSF) analysis. The result suggests malignancy comprising tumor cells with pleomorphic nuclei.

Medulloblastoma was diagnosed on the basis of initial presentation, therapeutic response, and multifocal recurrences. The patient was scheduled for radiotherapy and chemotherapy. Craniospinal irradiation of 36 Gy was performed in 33 fractions for 2 months, with
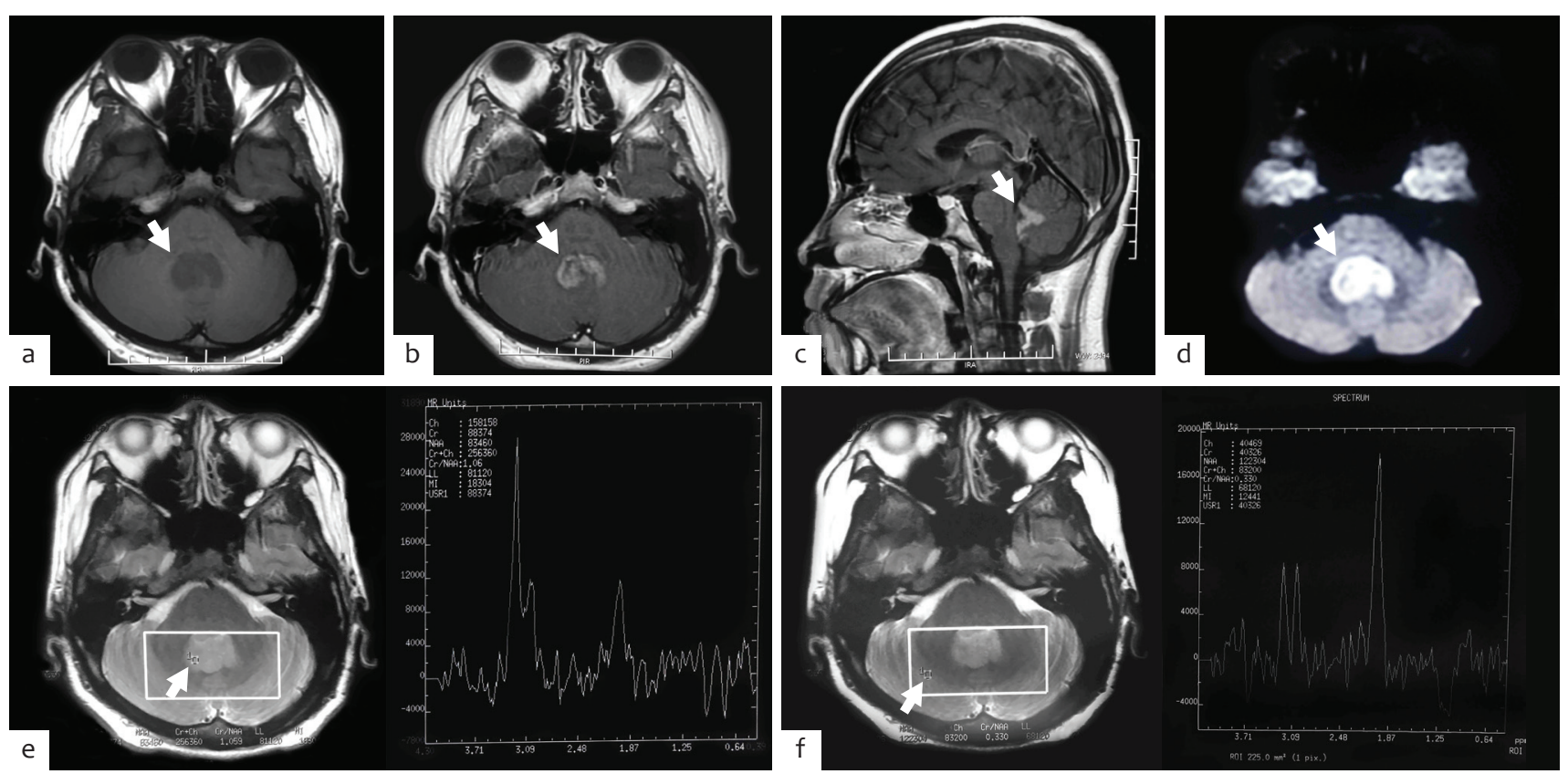

Figure 1. Initial brain magnetic resonance (MR) and MR spectroscopy presentation in the first meeting. MR images at presentation showed hypointense cerebellar vermis mass (arrows) on T1 weighted image (a), with heterogeneous enhancement (b and c). Diffusion-weighted imaging showed an area of hyperintensity (arrow) (d). No signs of obstructive hydrocephalus. Single-voxel spectroscopy of the mass (arrow) (e) showed a higher Cho/NAA ratio of 1.89 compared with that of the normal cerebellar parenchyma (arrow) (f) with 0.33

Cho=Choline; $\mathrm{NAA}=\mathrm{N}$-acetyl-aspartate

mji.ui.ac.id 

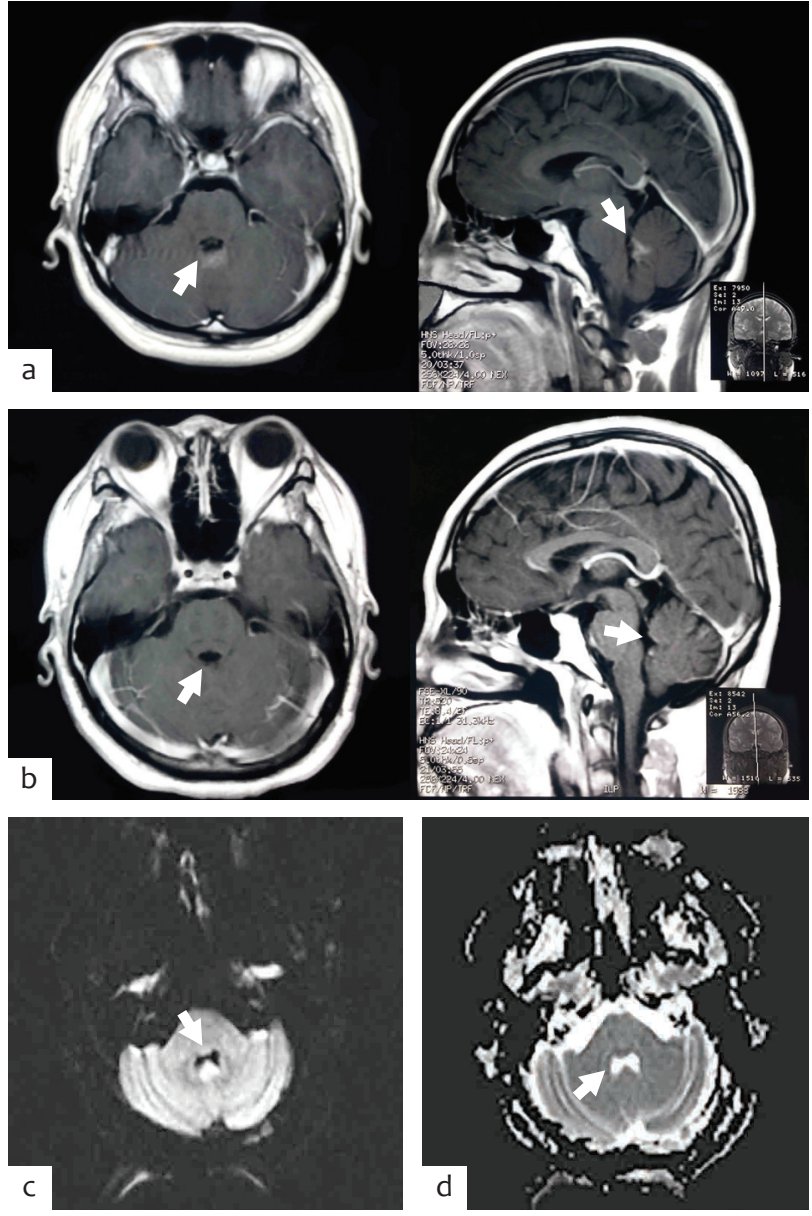

Figure 2. Brain magnetic resonance following cerebellar vermis stereotactic radiotherapy. Axial and sagittal postcontrast images showing volume reduction of enhancing mass (arrows) located posterior to the fourth ventricle 1 month following complete radiation (a), with complete disappearance of the tumor 5 months following radiation (b). Axial diffusion-weighted imaging (c) and apparent diffusion coefficient map (d) obtained 5 months after irradiation showed no pathological diffusion restriction of the cerebellar vermis (arrows)

local booster up to 56 Gy for brain lesions, 50 Gy for cervical lesions, and 60 Gy for lumbosacral lesions. The chemotherapy regimen consisted of four cycles of vincristine followed by six cycles of carboplatin and etoposide.

After completing the therapy regimen, spinal and brain MR was performed in $8^{\text {th }}$ month after the previous follow-up to evaluate therapeutic response (Figure $3 \mathrm{e}-\mathrm{j}$ ). Brain MR depicted size reduction of the enhancing suprasellar mass in the pituitary stalk, with loss of small lesions in the cerebral sulci. Single-voxel suprasellar MRS showed an integral Cho value of 26.6, an NAA value of 31.1, and a Cho/NAA ratio of 0.85 . Axial DWI and ADC map showed no pathological diffusion restriction of the pituitary stalk. Spinal MR revealed loss of multiple spinal lesions, such as the intradural $\mathrm{C}_{4}-\mathrm{C}_{5}$ lesion. The patient then underwent regular evaluation every 3 months to observe progression of the disease.

Brain MR follow-up was performed 13 months after completing radiotherapy and chemotherapy. The results showed an additional contrast-enhancing extra-axial lesion in the right temporal lobe (Figure 4). Suprasellar lesion was unchanged. The patient underwent SRT of 15 Gy targeted to the right temporal lobe mass. The patient received a regimen of oral temozolomide every 4 weeks.

Follow-up of brain MR approximately 2.5 years and 51 months after the previous MR examination showed further enlargement of the right temporal lobe lesion and consistent enhancement on the edges of its cystic components (Figure 5). DWI and ADC map showed pathological restriction diffusion. Multi-voxel MRS of the tumor showed a peak Cho value of 141,130; an NAA of 47,088; and a Cho/NAA ratio of 2.99, whereas MRS of the cerebral parenchyma showed a peak Cho of 38,500; an NAA of 107,280; and a Cho/NAA ratio of 0.36. Increased solid components and heterogeneous enhancement were observed along with the corresponding area of pathological diffusion restriction. No spinal metastatic residue was detected in subsequent contrast-enhanced spinal MR images (not shown).

Afterward, the temporal mass was further enlarged, causing subfalcine and transtentorial herniation. Decompressive craniectomy was performed to relieve intracranial pressure, and a simultaneous biopsy of the right temporal mass was conducted to confirm the diagnosis. Pathological results with hematoxylin and eosin (HE) staining showed densely packed small round blue cells that are distributed in a ribboning pattern with areas of necrosis, rosette formation, and mitosis. Ki-67 immunohistochemistry showed a high proliferation index of $40 \%$. Several immunohistochemistry staining results also showed that the tumor was synaptophysin positive, glial fibrillary acidic protein (GFAP) negative, and $\beta$-catenin negative. Following this finding, the patient received chemotherapy of cytarabine, followed by a cisplatinvincristine-cyclophosphamide regimen. The right temporal lobe lesion was seen enlarged following chemotherapy, causing only minimal symptoms. We concluded that the tumor was non-responsive toward further radiotherapy and systemic therapy. The patient give an consent for this publication. 

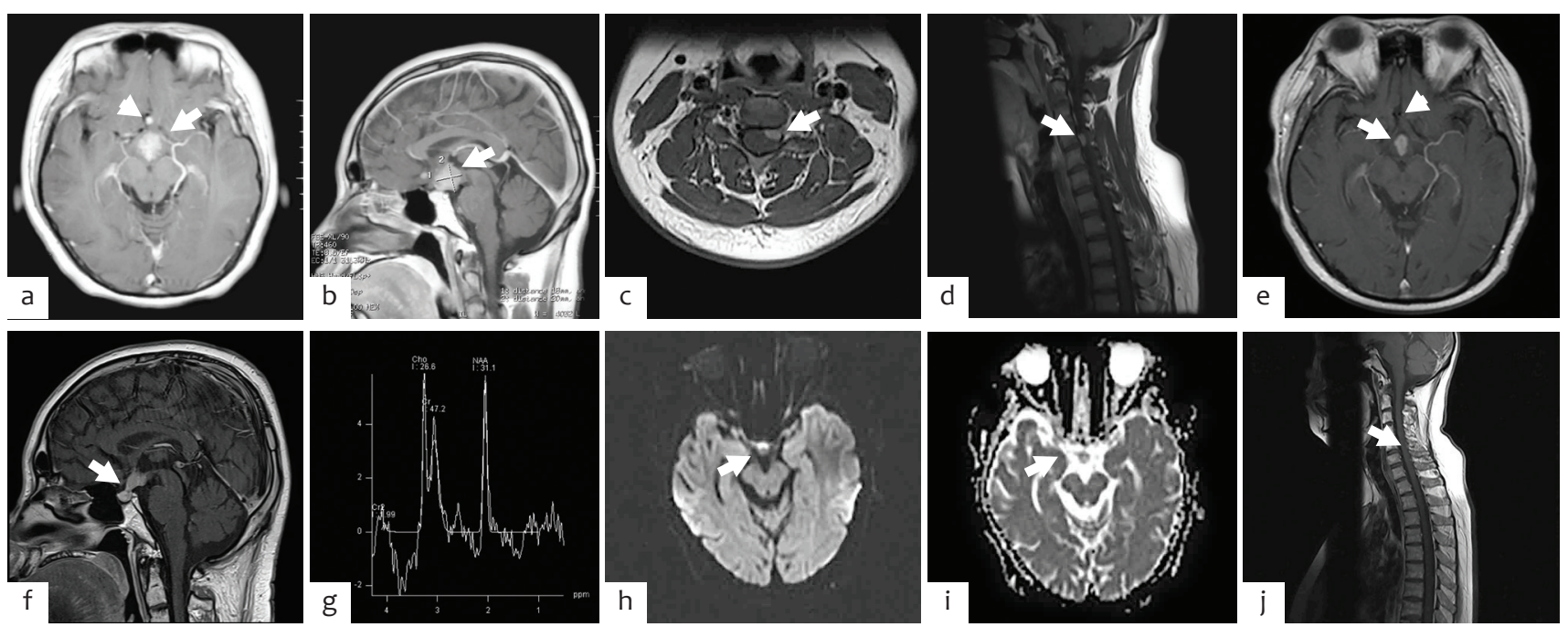

Figure 3. Brain and spinal MR performed 12 months following total disappearance of the cerebellar tumor (a-d) and after completing following radiation and chemotherapy (8 months after the previous examination) (e-j). Images showing vividly enhancing suprasellar mass (arrows) on post-contrast T1WI ( $a$ and b). Contrast-enhancing lesions were also observed in the interhemispheric sulci (arrowhead) (a) and intradural C4-C5 (arrows) (c and d) in post-contrast T1WI. After completing radiation and chemotherapy, axial and coronal images showed persistent enhancing pituitary stalk mass (arrows) with size reduction (e and f). Single-voxel MRS of the tumor showed a Cho/NAA integral ratio of 0.85 (g). Diffusion weighted imaging (arrow) (h) and apparent diffusion coefficient map (arrow) (i) showed no pathological diffusion restriction of the mass. At this point, no contrast-enhancing lesion was found in the interhemispheric sulci (arrowhead) (e) and intradural $\mathrm{C}_{4}-\mathrm{C}_{5}$ (arrow) (j)

$\mathrm{MR}=$ magnetic resonance; $\mathrm{T} 1 \mathrm{WI}=\mathrm{T} 1$ weighted image; $\mathrm{MRS}=\mathrm{MR}$ spectroscopy; Cho=Choline; $\mathrm{NAA}=\mathrm{N}$-acetyl-aspartate

\section{DISCUSSION}

This patient presented with prolonged headache which is a common presentation for medulloblastoma. According to Smoll et al, ${ }^{4}$ medulloblastoma can present in adolescents but is more often found in children. Headache, nausea, and vomiting in this patient could be caused by increased intracranial pressure. ${ }^{1}$ Although hydrocephalus was not observed in the imaging, the symptoms can precede the imaging presentation of hydrocephalus up to 2 months. ${ }^{5}$

A diagnosis of medulloblastoma should be considered based on the first radiological imaging. MR imaging showed heterogeneously enhancing mass of cerebellar vermis, hyperintensity on DWI, and an increase in Cho/NAA ratio. This feature is in line with the findings of Dhall et $\mathrm{al}^{1}$ and $\mathrm{O}^{\prime}$ Brien et $\mathrm{al}^{6}$ that medulloblastoma often presents as a midline mass with inhomogeneous enhancement. Perreault et $\mathrm{al}^{7}$ stated that off-midline medulloblastoma is rarely found, especially in older patients. DWI hyperintensity is related to restricted diffusion relative to the surrounding tissue caused by hypercellularity of the mass. ${ }^{6}$ Findings of the MRS appropriate with the results of Verma et $a^{8}$ that NAA reduction indicates axonal loss of the tumor and choline increase in cellularity and tumor cell turnover. ${ }^{8}$

The recurrence pattern for the case is suitable for medulloblastoma. Early recurrence usually occurs within
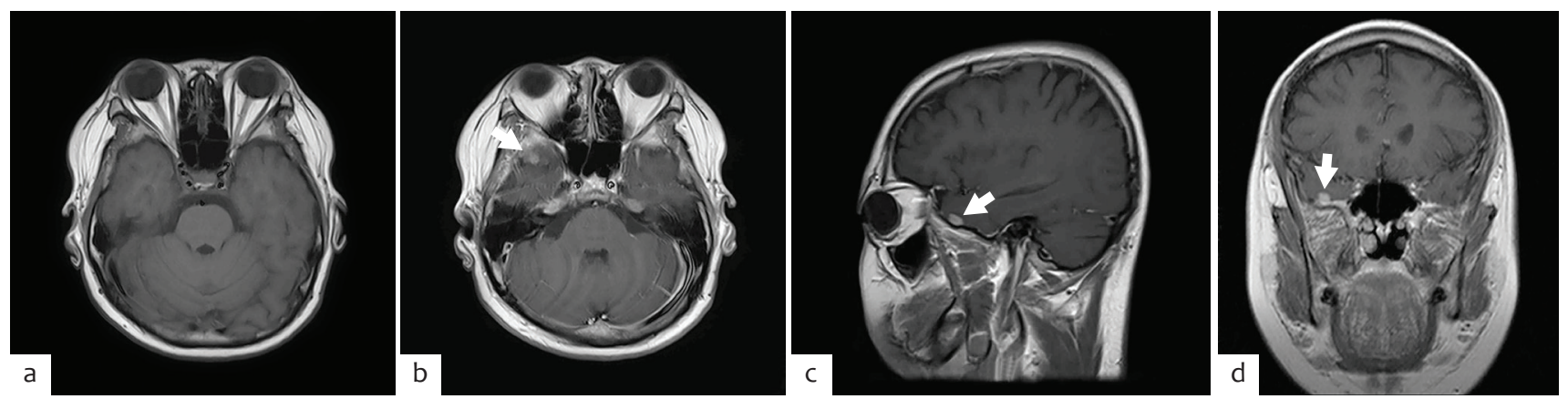

Figure 4. Brain magnetic resonance performed 13 months after completing radiation and chemotherapy. Isointense extra-axial right temporal lobe lesion in T1 weighted image (a), with vivid enhancement in post-contrast images (arrows) (b, c, and d) 

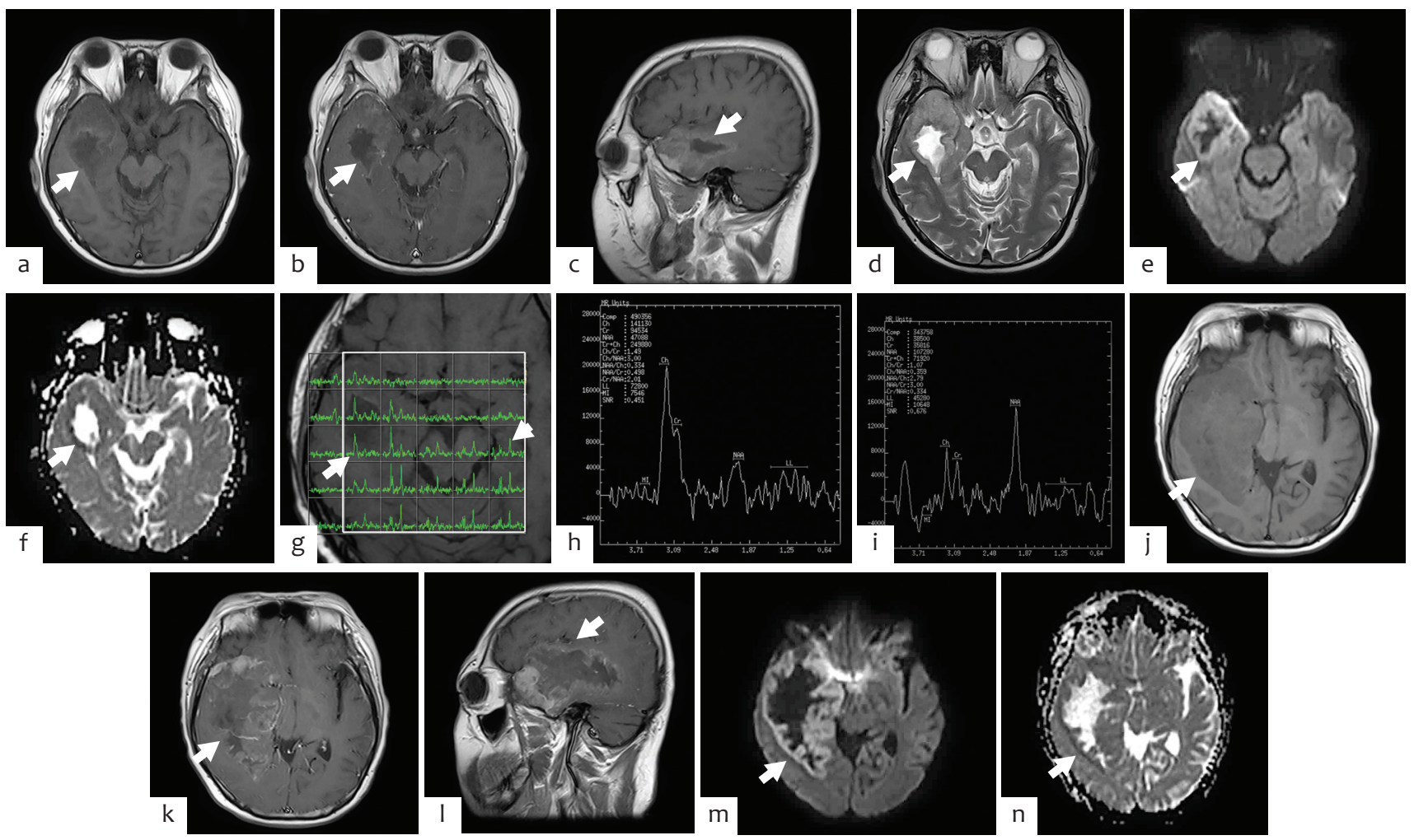

Figure 5. Follow-up brain MR and MRS performed 2.5 years $(\mathrm{a}-\mathrm{i})$ and approximately 4 years $(\mathrm{j}-\mathrm{n})$ after extra-axial temporal lesion was discovered. Temporal lobe mass (arrows) enlargement is observed in T1WI (a) and T2WI (d) with enhancement of the cystic component edges in post-contrast images (b and c). DWI (e) and ADC map ( $f$ ) confirmed pathological restriction diffusion, notably at the edges of the lesion (arrows). Spectral map (g) of multi-voxel MRS showed representative voxel at the tumor edges (open arrow) and normal cerebral parenchyma (arrowhead). MRS of the tumor (h) showed a higher Cho/NAA peak ratio of 2.99 compared with that of the normal cerebellar parenchyma (i) with 0.36. Further enlargement was observed in subsequent T1WI images (arrow) (j), with irregular inhomogeneous enhancement in post-contrast images (arrows) (k and I) and persistent peripheral pathological diffusion restriction (arrows) in DWI (m) and ADC map (n). Mass effect was shown by a subtle midline shift to the left $M R=$ angnetic resonance; $M R S=M R$ spectroscopy; $T 1 \mathrm{Wl}=\mathrm{T} 1$ weighted image; $\mathrm{T}_{2} \mathrm{~W}_{1}=\mathrm{T} 2$ weighted image DWl=diffusion-weighted

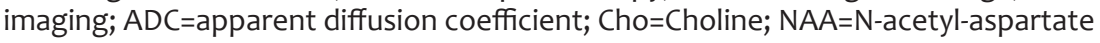
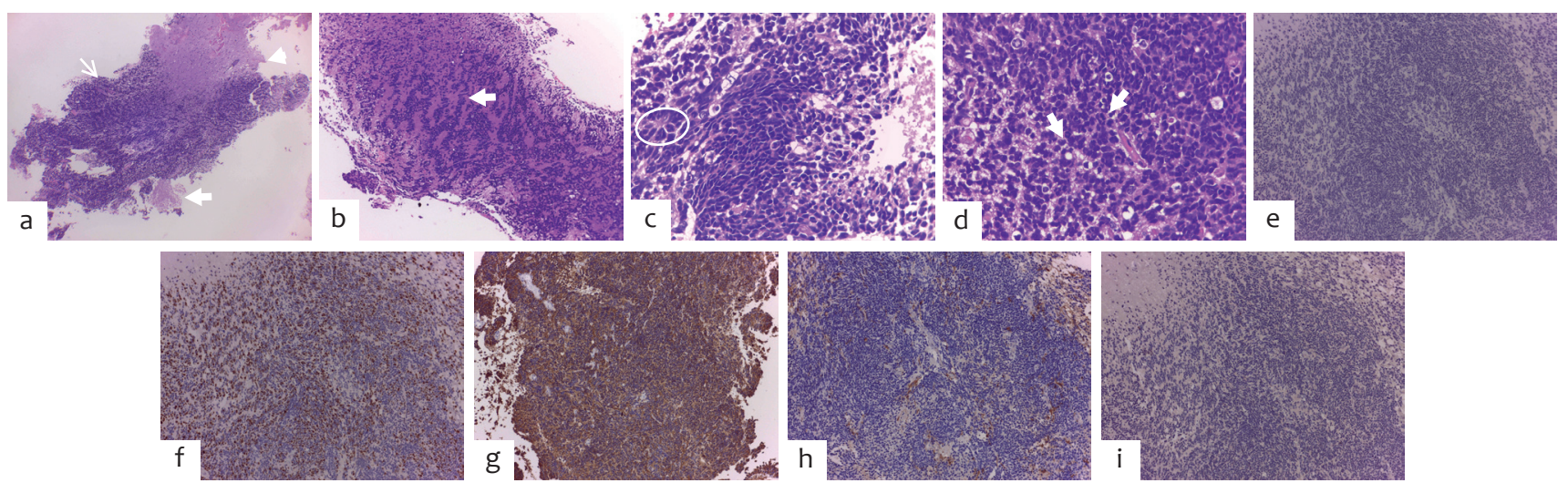

Figure 6. Pathological examination of the temporal lobe mass with HE staining (a-d) and immunohistochemistry ( $f-i)$. Lowpower photomicrograph shows tumor cells (arrow) infiltrating the brain parenchyma (arrowhead) with areas of necrosis (open arrow) (a). Low-power photomicrograph shows an area of tumor cells distributed in a ribboning pattern (arrow) (b). High-power photomicrograph (c and d) shows densely packed small round blue cells with the presence of Homer Wright rosette (circle in c) and mitosis (arrows in D). Low-power photomicrograph illustrates negative control for immunohistochemistry which is of purple color (e). Low-power photomicrograph from Ki-67 immunohistochemistry shows high mitotic activity with a proliferation index of $40 \%$, counted as number of positive (brown) cells from the total visible cells (f). Low-power photomicrograph of other immunohistochemical staining shows the tumor is synaptophysin positive being brown colored (g), GFAP negative (h), and $\beta$-catenin negative with both of them being purple colored similar to the negative control (i).

$\mathrm{HE}=$ hematoxylin and eosin; GFAP= glial fibrillary acidic protein 
2 years of post-treatment, ${ }^{1}$ in which our case showed recurrence 1 year after the loss of tumor residue was declared. Leptomeningeal dissemination tendency of medulloblastoma was clearly observed in this case, showing spinal and supratentorial seeding. Spinal metastatic deposit showed intradural extramedullary enhancing nodule, consistent with the statement of Ozerov et al. ${ }^{9}$ The presented case shown the presence of supratentorial seeding, although Lee et al, ${ }^{3}$ stated that supratentorial seeding of medulloblastoma less often. Each supratentorial lesion originated in the area with close contact to the CSF, both suprasellar and sulci area lesions. These findings support the concept that multiple metastatic lesions are related to the dissemination of medulloblastoma, in which all lesions start as extra-axial lesions.

Immunohistochemical staining revealed that the temporal lobe mass is synaptophysin positive, indicating that the tumor is composed of neuroendocrine cells, ${ }^{10}$ and GFAP negative, indicating that the tumor is of nonglial origin. ${ }^{11} \mathrm{HE}$ staining of the mass further limited the differential of the tumor to be of small round blue cells with a high proliferation index in the Ki-67 staining, which is consistent with medulloblastoma. ${ }^{10,12}$ The presence of Homer Wright rosette is pathognomonic for medulloblastoma in the posterior fossa tumor but can also be found in other supratentorial tumors, such as primitive neuroectodermal tumor and pineoblastoma. ${ }^{13}$ For the temporal lobe mass of the presented case, the presence of rosette formation when combined with other pathological information, such as densely packed small round blue cell tumor with areas distributed in a ribboning pattern in HE staining, is highly suggestive of classic variant medulloblastoma. ${ }^{12,13} \beta$-catenin staining was not performed to exclude other possible diagnoses but is useful in determining the medulloblastoma molecular subgroup; a negative result showed the tumor is non-wingless (WNT) activated. ${ }^{12}$

In the presented case, final MR imaging of the mass did not resemble typical medulloblastoma or its leptomeningeal metastasis. Although the patient's initial diagnosis of medulloblastoma could be mainly considered through the mass characteristics on the cerebellar vermis, the final MR presentation of the patient strongly showed its characteristic as an intra-axial mass of the temporal lobe without any clear trace of its leptomeningeal dissemination. MRS plays an important role in ruling out radiation necrosis, considering the alteration of Cho/NAA ratio and supported by progressive enlargement of the enhancing tumor area up to 4 years after SRT. ${ }^{1}$ A radiologist or pathologist with merely information regarding final imaging or its tumor tissue would erroneously diagnose the mass as a primary intraaxial mass, particularly with the absence of any mass within the cerebellum. Final proper diagnosis is only possible because of the long follow-up, which provides abundant prior information, such as the existence of prior cerebellar mass and multiple metastatic spinal seeding that disappeared after radiation and chemotherapy, and the initial lesion of the temporal lobe that could be observed as an extra-axial metastatic deposit (Figure 4). This knowledge suggests that the final presentation of intra-axial mass started in the area with a close CSF contact and then expanded toward brain parenchyma, compatible with the leptomeningeal seeding of primary tumor. ${ }^{1}$ Medulloblastoma has a high tendency to disseminate through the CSF whether as a spinal deposit or supratentorial seeding. ${ }^{13,9}$ Therefore, the final diagnosis of metastatic seeding of the temporal lobe of medulloblastoma was made in accordance with prior data of cerebellar tumor, information regarding multiple spinal and supratentorial metastatic seedings, the initial deposit of the temporal lobe, and histological findings.

Multiple radiological information combined with pathological results could aid in estimating the molecular subgroup of medulloblastoma. The four main subgroups consisted of WNT-activated, sonic hedgehog (SHH)activated, group 3, and group 4. In terms of initial tumor location, the SHH-activated subgroup occurs at the cerebellar hemispheres, the WNT-activated subgroup occurs at the cerebellopontine angle, and groups 3 and 4 occur at midline. ${ }^{7}$ Recurrence patterns show that the $\mathrm{SHH}$-activated subgroup has local recurrences, groups 3 and 4 have recurrences outside the original tumor bed, and the WNT-activated subgroup could have either local or metastatic recurrence.7 Regarding the histological findings, classical medulloblastoma showing small blue round cells with Homer Wright rosettes is dominantly found in groups 3 and 4, whereas desmoplastic medulloblastoma showing densely packed undifferentiated cells with hyperchromatic and pleomorphic nuclei are commonly found in the $\mathrm{SHH}$ activated subgroup. Although groups 3 and 4 are similar in characteristics, contrast-enhanced MR could aid the differentiation, in which group 4 would show minimal to none enhancement of the mass. The presented case was initially shown as a midline enhancing mass of the 
cerebellar vermis, which then spread through the CSF into the spinal and supratentorial deposits without tumor bed recurrence. In addition, histological findings of the temporal lobe were consistent with classic medulloblastoma. Supported with the information that the medulloblastoma subgroup would not change during recurrences, group 3 is the most likely molecular subgroup of the presented case.7,14

Considering our report, clinicians should encourage routine examination and MR imaging of post-treatment medulloblastoma patients. If local recurrence or distant metastasis develops, the availability of multiple imaging data during routine follow-up would greatly aid to determine the correct diagnosis. Surgical resection of the cerebellar mass should be strongly suggested. Even though medulloblastoma showed favorable response to external radiation and chemotherapy, the principal treatment of medulloblastoma is surgical resection. ${ }^{15,16}$ This case showed the poor outcome of medulloblastoma that did not undergo surgical resection, which later showed multiple leptomeningeal disseminations despite adequate radiation and chemotherapy.

\section{Conflicts of Interest}

The authors affirm no conflict of interest in this study.

\section{Acknowledgment \\ None. \\ Funding Sources \\ None.}

\section{REFERENCES}

1. Dhall G. Medulloblastoma. J Child Neurol. 2009;24(11):1418-30. 2. Kumar S, Handa A, Jha DK, Choudhary A. Supratentorial metastasis of medulloblastoma in adults. Asian J Neurosurg. 2016;11(3):320.

3. Lee DS, Cho J, Kim SH, Kim DS, Shim KW, Lyu CJ, et al. Patterns of failure following multimodal treatment for medulloblastoma: long-term follow-up results at a single institution. Cancer Res Treat. 2015;47(4):879-88.

4. Smoll NR, Drummond KJ. The incidence of medulloblastomas and primitive neurectodermal tumours in adults and children. J Clin Neurosci. 2012;19(11):1541-4.

5. Krishna V. Textbook of pathology. 1st ed. Hyderabad: Orient Longman Private Limited; 2004.

6. O'Brien WT. Imaging of primary posterior fossa brain tumors in children. J Am Osteopath Coll Radiol. 2013;2(3):2-12.

7. Perreault S, Ramaswamy V, Achrol AS, Chao K, Liu TT, Shih D, et al. MRI surrogates for molecular subgroups of medulloblastoma. Am J Neuroradiol. 2014;35(7):1263-9.

8. Verma A, Kumar I, Verma N, Aggarwal P, Ojha R. Magnetic resonance spectroscopy-revisiting the biochemical and molecular milieu of brain tumors. BBA Clin. 2016;5:170-8.

9. Ozerov SS, Tereshchenko GV, Artemov AV, Kumirova EV, Samarin AE, Papusha LI, et al. MRI diagnosis of spinal metastasis of medulloblastoma. A clinical case and literature review. Zh Vopr Neirokhir Im N N Burdenko. 2015;79(5):72-6.

10. Meurer RT, Martins DT, Hilbig A, de Castro Ribeiro M, Roehe AV, Barbosa-Coutinho LM, et al. Immunohistochemical expression of markers Ki-67, neun, synaptophysin, p53 and HER2 in medulloblastoma and its correlation with clinicopathological parameters. Arq Neuropsiquiatr. 2008;66(2B):385-90.

11. Yang Z, Wang KK. Glial fibrillary acidic protein: from intermediate filament assembly and gliosis to neurobiomarker. Trends Neurosci. 2015;38(6):364-74.

12. Shih RY, Koeller KK. Embryonal tumors of the central nervous system: from the radiologic pathology archives. Radiographics. 2018;38(2):525-41.

13. Wippold FJ 2nd, Perry A. Neuropathology for the neuroradiologist: rosettes and pseudorosettes. AJNR Am J Neuroradiol. 2006;27(3):488-92.

14. Ramaswamy V, Remke M, Bouffet E, Faria CC, Perreault S, Cho YJ, et al. Recurrence patterns across medulloblastoma subgroups: an integrated clinical and molecular analysis. Lancet Oncol. 2013;14(12):1200-7.

15. Martin AM, Raabe E, Eberhart C, Cohen KJ. Management of pediatric and adult patients with medulloblastoma. Curr Treat Options Oncol. 2014;15(4):581-94.

16. Parkes J, Hendricks M, Ssenyonga P, Mugamba J, Molyneux E, Schouten-van Meeteren A, et al. SIOP PODC adapted treatment recommendations for standard-risk medulloblastoma in low and middle income settings. Pediatr Blood Cancer. 2015;62(4):553-64. 